\title{
My child has smelly urine - questions
}

\author{
Peter Felix • Vijith Puthi • Mona Aslam
}

Received: 23 May 2011 /Revised: 8 September 2011 / Accepted: 9 September 2011 /Published online: 29 October 2011

(C) IPNA 2011

Keywords Smelly urine $\cdot$ Children

\section{Case summary}

A healthy 14-year-old boy presented to the outpatient department with a 6-year history of foul, fishy-smelling urine and body odor. He was being bullied at school, and his relatives had also noted the odor. He had no other urinary symptoms. His bowel habits were normal. Fluid intake was in excess of 6 cups of fluid per day and micturition was regular. There was no history of enuresis. Apart from being colorblind and having a few areas of hypopigmented patches around his elbow, his clinical examination was unremarkable.
Urine cultures sent from primary care and repeated in the clinic showed no bacterial growth. Urine tuberculosis culture was negative, and organic acid tests showed only mild lactic aciduria; amino acids were normal. Liver function, urea, and creatinine levels were within normal range.

Family members were well, with no significant illnesses; there was no history of renal disease. The young lad was not on medication and denied any drug involvement.

\section{Questions}

1. What are the causes of smelly urine?

2. What is the likely cause in this case? How would you confirm the diagnosis and treat the problem?

The answers to these questions can be found at http://dx.doi.org/ $10.1007 / \mathrm{s} 00467-011-2022-7$

P. Felix $\cdot$ V. Puthi $\cdot$ M. Aslam $(\bowtie)$

Department of Paediatrics Peterborough City Hospital,

Bretton Gate,

Peterborough PE3 9DA, UK

e-mail: mona.aslam@pbh-tr.nhs.uk 